\title{
Silent Hypoxia
}

\author{
Dhivagar Subbarayan ${ }^{1}$, Jasmine Farhana Mohamed Sheriff ${ }^{2}$
}

\begin{abstract}
COVID-19 caused by SARS-CoV-2 might be present with a wide range spectrum of symptoms from mild upper respiratory tract infection to severe pneumonia and may lead to death. COVID patients may experience severe hypoxia without consistent features of respiratory distress; this is also called silent/happy/apathetic hypoxia. The exact mechanism of happy hypoxia is unknown; however, different assumptions include that COVID-19 idiosyncratic effect may damage the respiratory control system and cause severe damage to the lungs, and may lead to the sudden collapse of the lungs with the evidence of sudden dropout of $\mathrm{SpO}_{2}$. Hence, it is a serious life-threatening condition that can need immediate medical attention.
\end{abstract}

Keywords: Apathetic hypoxia, Dyspnea, Silent hypoxia.

Pondicherry Journal of Nursing (2021): 10.5005/jp-journals-10084-13111

\section{INTRODUCTION}

Silent hypoxia is also known as happy hypoxia. Hypoxia is defined as a very low oxygen level in the blood. In a healthy person, 95 to $100 \%$ is considered normal blood oxygen saturation; in COVID-19 patients, the $\mathrm{SpO}_{2}$ level shows dangerous declines of less than $40 \%$. The warning vital sign is hypoxia and it shows usual symptoms of shortness of breath; happy hypoxia cannot show any external signs in a healthy individual. At the initial stage, a sick COVID-19 patient is asymptomatic and appears to be all right and happy. Happy hypoxia is an entity that describes the objective of hypoxia in the absence of warning signs and symptoms of upper respiratory distress that is proportionate to the findings of the current patient scenario. ${ }^{1}$

\section{INCIDENCE}

Silent hypoxia can affect both genders and it occurs in every 10 patients usually between two and four experience silent hypoxia with collapse of the lungs. A medical emergency is needed when a patient is having dropout of $\mathrm{SpO}_{2}$ and if he/she experiences COVID symptoms for the past 5-7 days, and during the period of signs and symptoms of COVID-19, sudden fluctuating oxygen saturation may lead to a life-threatening situation. ${ }^{1}$

\section{Prevalence of Silent Hypoxemia in COVID-19}

Busana and Gasperetti ${ }^{2}$ conducted a retrospective observational cohort study in a population consisting of 213 COVID-19 patients and the findings show that silent hypoxemia is remarkably present in COVID-19. The presence of dyspnea is associated with a more severe clinical condition. When the chest X-ray was severely abnormal, $25 \%$ of the population was silent hypoxemic. Also, 26.5 and $38.6 \%$ of silent and dyspneic patients were admitted to the ICU, respectively $(p=0.082)$. The mortality rate was 17.6 and $29.7 \%$ (log-rank $p=0.083)$ in silent and dyspneic patients. ${ }^{2}$

\section{Physiology of Silent Hypoxia}

Silent hypoxia is one of the known complications of COVID-19. It is speculated that this life-threatening condition is caused by

\footnotetext{
1,2Department of Psychiatric Nursing, Kasturba Gandhi Nursing College, Sri Balaji Vidyapeeth, Puducherry, India

Corresponding Author: Dhivagar Subbarayan, Department of Psychiatric Nursing, Kasturba Gandhi Nursing College, Sri Balaji Vidyapeeth, Puducherry, India, e-mail: dhivagalaxy@gmail.com

How to cite this article: Subbarayan D, Sheriff JFM. Silent Hypoxia. Pon J Nurs 2021;14(4):86-87.

Source of support: Nil

Conflict of interest: None
}

SARS-CoV-2, directly affecting the blood flow to the lung airways; it can collapse the lung oxygen sufficiency but is not affecting them enough to cause shortness of breath. The formation of small blood clots within the lungs also may cause a dropout in the saturation rate. It is also shown that the breathing level of patients with COVID-19 gradually increases, which in turn leads to happy hypoxia. COVID-19 patients experience lesser levels of shortness of breath but suddenly the oxygen saturation starts to drop out and reach the oxygen insufficiency state and the patient needs immediate $\mathrm{O}_{2}$ support. ${ }^{3}$

\section{Diagnosis}

A six-minute walk test is one of the easiest ways to identify the oxygen stability and saturation level in the blood. Instructed patients to walk continuously for 6 minutes. A pre-check $\mathrm{SpO}_{2}$ has been done and the post-check $\mathrm{SpO}_{2}$ was also checked once completing the walk test in order to monitor their physiological response. The results showed that after the completion of 6 minutes of walk COVID-19 patients were more likely to develop exerciseinduced hypoxia without symptoms comparing to non-COVID-19 patients who suffered from idiopathic symptoms of pulmonary fibrosis. This walk test may be a diagnostic test and may help identify preventive modalities of silent hypoxia. ${ }^{4}$

\section{Prognosis}

The prognosis of happy hypoxia is poor. Decreased levels of oxygen saturation below the range of $50 \%$ may cause serious lifethreatening situations and affect the recovery of a patient. There are

(c) The Author(s). 2021 Open Access This article is distributed under the terms of the Creative Commons Attribution 4.0 International License (https://creativecommons. org/licenses/by-nc/4.0/), which permits unrestricted use, distribution, and non-commercial reproduction in any medium, provided you give appropriate credit to the original author(s) and the source, provide a link to the Creative Commons license, and indicate if changes were made. The Creative Commons Public Domain Dedication waiver (http://creativecommons.org/publicdomain/zero/1.0/) applies to the data made available in this article, unless otherwise stated. 
three reasons for COVID-19 patients to get silent hypoxia. Hypoxia has the ability to damage the respiratory system and create oxygen insufficiency without showing any clinical symptoms. In coronavirus patients, it can first damage the lungs and can cause a sudden dropout of $\mathrm{O}_{2}$ saturation.

- Airway obstruction may cause dropout of $\mathrm{SpO}_{2}$

- More production of carbon dioxide in the blood could cause heavy breathing to exhalation and collapse the lung parenchyma.

- Respiratory diseases may cause a decrease in lung compliance. ${ }^{5}$

\section{Gasping for Air}

Normal oxygen saturation level is around $95-100 \%$. It starts to become worse and creates warning signals at levels below $90 \%$. Sufficient oxygen cannot be supplied to the central nervous system and patients might start experiencing mental dysfunctions like confusion, clogging, and disorientation and difficulty in breathing, and as the $\mathrm{SpO}_{2}$ level drops into the low $80 \%$ or below, it starts to show the danger of damage to the vital organs. ${ }^{6}$

\section{Prevention}

Identifying happy hypoxia in COVID-19 patients before they start to experience shortness of breath is critical for preventing pneumonia from progressive deterioration. The prevention of this condition is to identify the initial dropout of oxygen saturation in COVID-19 patients who start to suffer from pneumonia in the early stage of illness and start the medical management to prevent the lungs from deteriorating to collapse. At the same time, early detection of COVID-19 pneumonia helps prevent a patient from invasive procedures, such as intubation and mechanical ventilation, this life-saving procedure, which is currently shown in an $80 \%$ of the mortality rate for COVID-19 patients. ${ }^{6}$

\section{Geriatric Considerations}

Geriatric is a more vulnerable population. Medical attention is needed for a dropout of saturation rate and keen observation of $\mathrm{O}_{2}$ saturation of the elderly daily may prevent silent hypoxia. Old age with comorbidities may add to the risk factor for this life-threatening condition. Hence, we should maintain the $\mathrm{O}_{2}$ saturation at a normal state which can lead to a healthy lifestyle. ${ }^{6}$

\section{ImPACt Of Silent Hypoxia}

Silent hypoxia is a life-threatening condition in COVID-affected patients. Without arising initial clinical symptoms, it may cause serious health issues. Youth and adolescents may recover with initial management and the most considering part is the elderly with comorbidities. Continuous monitoring of $\mathrm{O}_{2}$ saturation may be the key source to detect this condition. Initial awareness is needed to avoid anxiety about this condition. Boosting up your oxygen saturation level naturally and doing daily healthy practices may help prevent this happy hypoxia. ${ }^{7}$

\section{Conclusion}

Silent hypoxia is a life-threatening medical condition that needs serious medical attention at an early stage of illness that may prevent the mortality rate. Hence, continuous monitoring of COVID positive patients' $\mathrm{O}_{2}$ saturation could prevent the severity of illness and can facilitate the primary preventive modalities. Hence, this study recommended and focused more on research on this clinical condition to prevent the life-threatening situation.

\section{References}

1. LaMotte S. Silent hypoxia: Covid-19 patients who should be gasping for air but aren't. CNN. May 2020.

2. Busana $M$, Gasperetti A. Prevalence and outcome of silent hypoxemia in COVID-19. Minerva Anestesiol 2021;87(3):325-333. DOI: 10.23736/ S0375-9393.21.15245.

3. Pappas S. "Silent hypoxia" may be killing COVID-19 patients. But there's hope. Live Sci 2020.

4. Jones R. Three reasons why COVID-19 can cause silent hypoxia. Sci Daily 2020.

5. Emily H. Silent hypoxia and its role in COVID-19 detection. News Med 2020.

6. Rouqui $P$, Amrane S. Asymptomatic hypoxia in COVID-19 is associated with poor outcome. Int J Infect Dis 2021;102:233-238. DOI: 10.1016/j. ijid.2020.10.067.

7. Bowden. What is "silent hypoxia"? The corona virus symptom patients don't know they have. Global News 2020. 